Abstract

\title{
Study of the Retrotransposon-Derived Human PEG10 Protease $^{+}$
}

\author{
Mária Golda ${ }^{1,2, *}$, János András Mótyán ${ }^{1}$, Mohamed Mahdi ${ }^{1}$ and József Tőzsér ${ }^{1}$ \\ 1 Department of Biochemistry and Molecular Biology, Faculty of Medicine, University of Debrecen, \\ 4032 Debrecen, Hungary; golda.maria@med.unideb.hu (M.G.); motyan.janos@med.unideb.hu (J.A.M.); \\ mohamed@med.unideb.hu (M.M.); tozser@med.unideb.hu (J.T.) \\ 2 Doctoral School of Molecular Cell and Immune Biology, University of Debrecen, 4032 Debrecen, Hungary \\ * Correspondence: golda.maria@med.unideb.hu \\ † Presented at Viruses 2020-Novel Concepts in Virology, Barcelona, Spain, 5-7 February 2020. \\ Published: 1 July 2020
}

\begin{abstract}
Paternally expressed gene 10 (PEG10) is a human retrotransposon-derived imprinted gene. Previous works have demonstrated that a mutation in the coding sequence of this gene is lethal with regard to embryological age due to defects of placental development. In addition, PEG10 is implicated in several malignancies, such as pancreatic cancer and hepatocellular carcinoma. The PEG10 gene encodes two protein isoforms, which are translated by a typical retroviral frameshift mechanism. The Gag-like protein (RF1PEG10) is encoded by reading frame 1, whilst reading frames 1 and 2 accounts for the Gag-Pol-like polyprotein (RF1/RF2PEG10). The protease (PR) domain of RF2PEG10 contains an -Asp-Ser-Gly- sequence, which refers to the conservative -Asp-Ser/Thr-Gly- active-site motif of retroviral aspartic proteases. The function of the aspartic protease domain of RF2PEG10 remains unclear. In order to further investigate the function of the PEG10 protease (PRPEG10), a frameshift mutant was generated (fsRF1/RF2PEG10) for comparison with the RF1/RF2PEG10 form. To study the effects of PRPEG10 on cellular proliferation and viability, mammalian HEK293T and HaCaT cells were transfected with plasmids encoding for either the frameshift mutant ( $\mathrm{fs}_{\mathrm{s}} \mathrm{RF} 1 / \mathrm{RF} 2 \mathrm{PEG}$ ) or a PR active-site (D370A) mutant fsRF1/RF2PEG10. Based on our findings, an fsRF1/RF2PEG10 overexpression resulted in an increased cellular proliferation, compared to the mutant form. Interestingly, transfection with fsRF1/RF2PEG10 had a detrimental effect on cell viability. We hypothesize that PRPEG10 may play a cardinal role in the function of this retroviral remnant, possibly implicated in cellular proliferation and the inhibition of apoptosis.
\end{abstract}

Keywords: PEG10; paternally expressed gene 10; cell viability; cell proliferation; cis protease activity; ubiquitination; homology modeling; retroviral-like protease; protease; retrotransposon

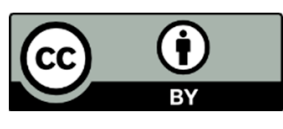

(C) 2020 by the authors. Licensee MDPI, Basel, Switzerland. This article is an open access article distributed under the terms and conditions of the Creative Commons Attribution (CC BY) license (http://creativecommons.org/licenses/by/4.0/). 